\title{
Improved Nucleation and Transition by Plasma Treatments for Fast Response Optically-Compensated-Bend Displays
}

\author{
G.M. Wu*, C.J. Huang And H.W. Chien \\ Institute of Electro-Optical Engineering, Green Technology Research Center, Chang Gung University \\ Kweisan, Taoyuan 333, Taiwan R.O.C.
}

\begin{abstract}
The optically-compensated-bend mode pi-cell displays exhibit fast-response time and wide-viewing angle characteristics. However, it requires a transition of the liquid crystal molecule from an initial splay state to the bend state configuration before providing the quick operation. A high voltage and a long warm-up time are needed to transform to the bend state. In this paper, the polyimide alignment films have been modified to reduce the splay-to-bend transition time by plasma beam treatments. The proposed method was demonstrated to be highly effective in improving the overall transition time. The number of splay-to-bend nucleation sites in the assembled liquid crystal cells could be increased dramatically by up to 20 times at the initial stage, and the improvement in the cell warm-up time was achieved at $45-71 \%$ reduction at $5.5 \mathrm{~V}$. The plasma processing parameters were optimized at the plasma power of $700 \mathrm{~W}$, the plasma distance of $25 \mathrm{~mm}$, and the plasma scan speed of $600 \mathrm{~mm} / \mathrm{s}$. In addition, we maintained the excellent optical properties and response time characteristics for the optically-compensated-bend mode liquid crystal displays.
\end{abstract}

DOI: $10.12693 /$ APhysPolA.123.892

PACS: 52.77.-j, 81.65.-b, 42.79.Kr, 64.60.Q-

\section{Introduction}

The rapid advancement in liquid crystal displays (LCD) led to the characteristics of low driving voltage, low power consumption, light weight, low production cost, and no hazardous radiation. The LCD devices are thus widely employed as television sets and computer monitors. They have become more popular than the traditional cathode-ray-tube (CRT) monitors nowadays. However, the conventional twist-nematic (TN) mode LCDs exhibit a viewing angle problem that results in the degradation of contrast ratio or gray scale inversion, and they turn out to be unsuitable for displaying motion pictures because of their slow response speed. The optically-compensated-bend (OCB) mode LCD displays have been introduced as electro-optical binary switching devices that offer wide viewing angle and fast response speed [1-4]. They also maintain good electro-optical properties at low operating temperatures. The stable gray scale could be achieved by introducing a bias voltage and wider viewing angle by optical compensation using a biaxial-retardation film.

The pi $(\pi)$ cell is constructed using a pair of substrates that are rubbed in parallel directions. When driving a $\pi$ cell in the bend state, the OCB mode liquid crystal molecules in the cell center experience zero elastic torque during the off-state relaxation process, thus providing a fast display response [5]. The response time can be 10-times faster than the traditional TN-LCD. It provides alternative solution to optical devices requiring quick re-

*corresponding author; e-mail: wu@mail.cgu.edu.tw sponse such as three-dimensional (3D) displays with high frequency or frame rate. On the other hand, OCB mode requires a transition of the liquid crystal cell from an initial splay state to bend state configuration before it can provide the quick operation. The transit voltage is inevitably high, and it takes a long warm-up time to transform to the bend state. This is a major disadvantage of the OCB mode displays. It is therefore desired to reduce the warm-up time. A splay-to-bend transition in the OCB cell is produced in two steps: the initial formation of bend nuclei and the eventual growth to the full bend state. The bend nucleation is uncertain and difficult to control, and the transition area grows slowly [6]. The purpose of this study is therefore to investigate the improved creation of nuclei and the bend transition by suitable surface plasma treatments of the alignment films.

In the earlier studies, tiny dark regions were observed to represent irregularities that occurred in liquid crystal alignment due to the existence of spacers. The regions acted as nuclei for the bend transition [7]. The high number of transition nuclei might accelerate the efficiency of the bend transition in the OCB display cells. In order to promote a faster splay-to-bend transition, we introduced the partial irregularity in the liquid crystal alignment by atmospheric pressure plasma beam treatments. The effects on the increase in the number of bend nucleus sites were evaluated using the $\pi$ cells constructed by a pair of polyimide (PI) alignment film substrates rubbed in parallel angles $[8,9]$. The overall transition time has been significantly reduced by the plasma beam bombardments of the PI alignment films. The OCB cell electro-optical properties were studied by the NI-DAQpad-6070E system equipped with function generators and LabVIEW7. In addition, the atomic force microscope (AFM, Veeco 
INNOVA) and a contact angle meter (FTA-25) were used to analyze the surface characteristics of the PI films both before and after the plasma beam treatments.

\section{Experimental}

The experiments were carried out on the glass substrates (Corning 1747) with the tin-doped indium oxide (ITO) coatings of the thickness of $100 \mathrm{~nm}$ by sputtering. After cleaning, they were cut into the size of $2 \times 2.5 \mathrm{~cm}^{2}$. The horizontal alignment PI (Chisso) material was spread using the SWIENCO PM490 spin-coater in two stages. The horizontal alignment layer had a nominal high pre-tilt angle of $6^{\circ}$ with the prescribed processing procedures. In the first stage, the spinning speed was set at $500 \mathrm{rpm}$ (round per min) for $10 \mathrm{~s}$, so that the alignment layer material could be evenly coated. In the second stage, the spinning speed was $2500 \mathrm{rpm}$ for $50 \mathrm{~s}$ to achieve a consistent thickness. A hot plate was used for soft bake at $80^{\circ} \mathrm{C}$ for $60 \mathrm{~s}$ to evaporate the excess water and solvent. The final hard bake was performed at $230^{\circ} \mathrm{C}$ for $30 \mathrm{~min}$, and the cured PI layer had a thickness of $80 \mathrm{~nm}$. The following mechanical rubbing of the alignment layer was conducted by the Skiing Tech RM50 rubbing system. The average rubbing speed was $20 \mathrm{~mm} / \mathrm{s}$, the speed of the roller was set at $500 \mathrm{rpm}$, the rubbing depth was $0.45 \mathrm{~mm}$, and the rubbing angle was zero.

Plasma treatment could be an effective method to modify polymeric material surface such as the PI alignment layer. The surface modification and activation are achieved by chemical and physical interactions of plasma with the surface molecules. The proposed atmospheric plasma treatment (APT) process used NEMS Tech Jet2008I that offered unique advantages for surface treatments. The APT system produced high-density plasma, and could provide treatments for various material substrates at low temperatures. The various processing parameters included plasma power $(W)$, plasma-to-substrate distance $(d)$, substrate scan speed $(v)$, and plasma bombardment angle. The plasma beam treatment experimental setup for the polyimide alignment layer to improve the OCB bend nucleus transition is schematically illustrated in Fig. 1. The surface energy level and/or characteristics could thus be optimized for the $\pi$ cell displays.

Finally, we used the UV-curable seal resin to assemble the LCD cell samples, and injected them with OCB (Merck GVD-100A) liquid crystal. The cell gap was controlled using the ball spacer blended in the UV-curable resin. The bend nucleation and transition were then studied under the polarized laser light using an instant $5.5 \mathrm{~V}$ voltage. The corresponding response time and $V-T$ curves were measured by the optical instrument (Otsula LCD5100). In addition, the plasma power was set at $700 \mathrm{~W}$ for different atmospheric pressure plasma beam treatments for the PI alignment layers. The plasma-to-substrate distance was chosen at $25 \mathrm{~mm}$ to better induce the partial liquid crystal alignment irregularity

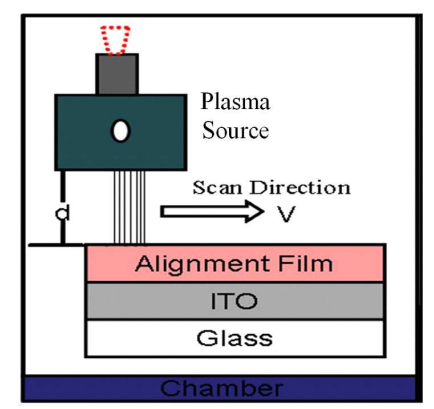

Fig. 1. Schematic illustration of the plasma beam treatment experimental setup for polyimide alignment layers.

and to increase the number of transition nucleus sites. A much longer distance created little plasma treatment effect, and a shorter distance has been proven to bring uneven damages to the samples. Nevertheless, the substrate scan speed has been varied at $550-650 \mathrm{~mm} / \mathrm{s}$ for optimal evaluation.

\section{Results and discussion}

Figure 2 shows the liquid crystal molecular alignment transition from the splay state to the bend state after being provided with an instantaneous voltage output of $5.5 \mathrm{~V}$ supply. Both the results of the OCB cell control sample (without plasma treatment) and the optimized plasma-treated sample $(v=600 \mathrm{~mm} / \mathrm{s})$ are provided for direct comparison. During the initial electric field-on period, some of the splay area transformed to bend transition nuclei in less than $10 \mathrm{~s}$ for the untreated cell sample in Fig. 2a. The number of nuclei was further increased and the bend state transition eventually covered the whole screen area after $92 \mathrm{~s}$. On the other hand, for the plasma-treated cells, the creation of bend nucleation was much faster. The dramatic increase in the number of bend nuclei was clearly evidenced in $7 \mathrm{~s}$ in Fig. $2 \mathrm{~b}$. The full transition to the bend state was also completed in $27 \mathrm{~s}$. This suggested the improvement of $71 \%$ in the reduction of the splay-to-bend transition time. The transition time data were measured and analyzed for different plasma treatment scan speeds at 650,600 and $550 \mathrm{~mm} / \mathrm{s}$, and are summarized in Table I. Therefore, the warm-up time for the OCB cells could be significantly reduced by $45-71 \%$ when compared with the control sample after the proposed atmospheric pressure plasma beam treatment process.

The higher number in transition nucleus accelerated the efficiency of liquid crystal bend transition, and thus reduced the splay-to-bend transition time in the OCB mode LCD cells. The effects of the plasma beam bombardment of the PI alignment film on the early-stage generation of the bend nucleus number are further depicted in Fig. 3. These photographs were taken only $6 \mathrm{~s}$ after the $5.5 \mathrm{~V}$ transition voltage was applied. The greatest number in the initial bend nucleus of 127 was 


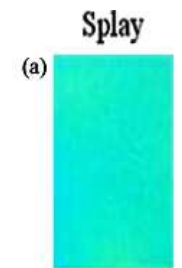

00:00

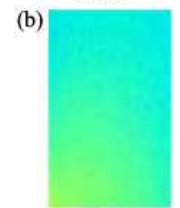

00:00

(l)

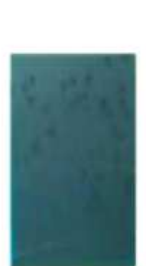

00:10

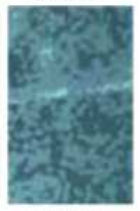

00:07

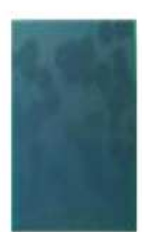

00:22

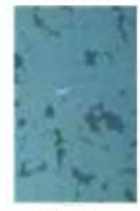

00:13

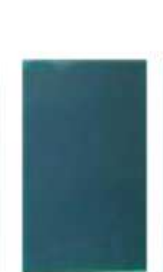

01:20

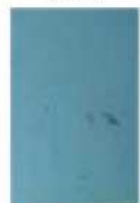

00:21
Bendl

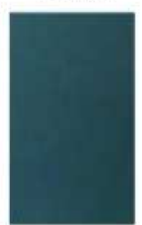

01:32

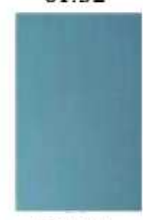

00:27

(3)
Fig. 2. OCB liquid crystal molecular alignment transition from splay state to bend state at $5.5 \mathrm{~V}$ : (a) control cell without plasma treatment and (b) the optimized plasma-treated sample at $600 \mathrm{~mm} / \mathrm{s}$.

TABLE I

OCB cell transition time data from splay to bend configuration. The warm-up time has been significantly reduced when compared with the control sample without the plasma treatment.

\begin{tabular}{l|c|c|c|c}
\hline \hline \multicolumn{1}{c|}{ Sample } & Control & $650 \mathrm{~mm} / \mathrm{s}$ & $600 \mathrm{~mm} / \mathrm{s}$ & $550 \mathrm{~mm} / \mathrm{s}$ \\
\hline transition time [s] & 92 & 51 & 27 & 49 \\
reduction [\%] & 0 & 45 & 71 & 47
\end{tabular}

found, and this is more than 20 times of that of the untreated control OCB cell. The measured data are also listed in Table II. It has been evidenced that the plasma scan speed of $600 \mathrm{~mm} / \mathrm{s}$ proved to produce the highest number of bend nuclei in the liquid crystal cell. This is quite consistent with the trend in the transition time data in Table I.
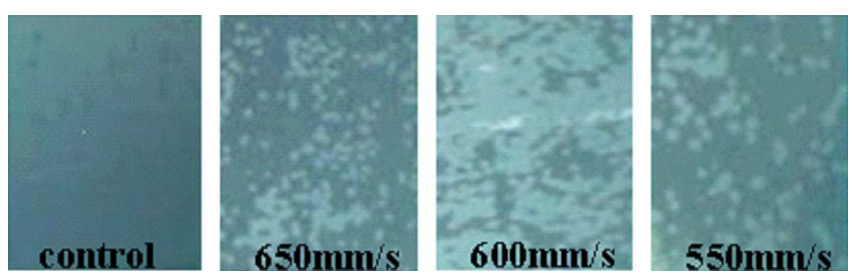

Fig. 3. Effects of plasma beam treatment scan speed on initial-stage of the generation of bend nucleus number. Six seconds after the $5.5 \mathrm{~V}$ transition voltage has been applied.

Figure 4 displays an example of the surface analysis result of the plasma-treated PI alignment film by AFM. The surface has been relatively smoothed with a low surface roughness (root-mean-square, RMS) of $0.28 \mathrm{~nm}$. No discernible morphological damage could be observed after the plasma beam treatment process. It remained uniform and flat. On the other hand, the contact angle analysis results are shown in Fig. 5. The room temperature con-
TABLE II

Measured bend nucleus number data from 0 to $6 \mathrm{~s}$ for the OCB cells.

\begin{tabular}{c|c|c|c|c}
\hline \hline Sample & Control & $650 \mathrm{~mm} / \mathrm{s}$ & $600 \mathrm{~mm} / \mathrm{s}$ & $550 \mathrm{~mm} / \mathrm{s}$ \\
\hline bend nucleus & 6 & 39 & 127 & 41
\end{tabular}

tact angle with de-ionized water was reduced from $86.0^{\circ}$ to $80.9^{\circ}$, indicating slight improvement in its hydrophilicity after the plasma treatment. The surface chemical functional groups have been activated and changed the chemical potentials.

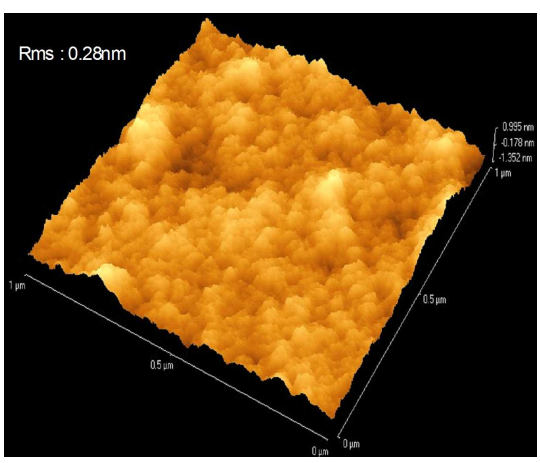

Fig. 4. AFM image of surface of plasma-treated PI alignment film. The surface has been relatively smoothed and no morphological damage was observed.

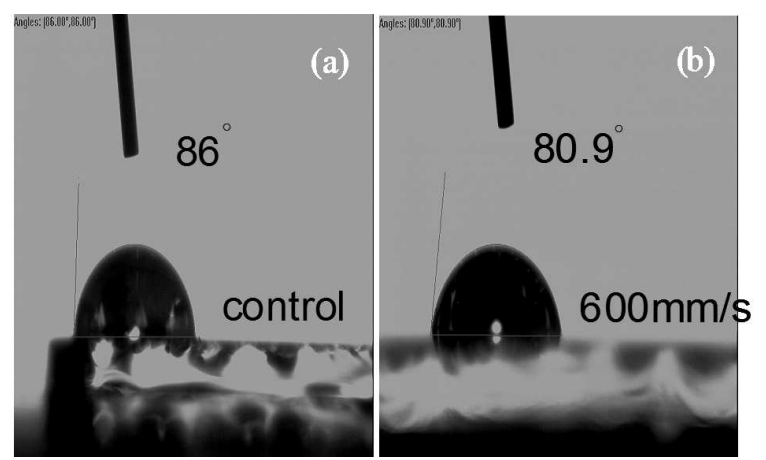

Fig. 5. Water contact angle analysis results: (a) control sample and (b) the optimized plasma-treated sample. A slightly more hydrophilic surface was found after plasma treatment.

The relationships between the transmittance and the applied voltage, $V-T$ curves, were measured for all the OCB cell samples. A square wave voltage with the frequency of $1 \mathrm{kHz}$ was employed, and the elevation rate of the applied voltage was $0.1 \mathrm{~V} / \mathrm{s}$. The electro-optical properties were measured with a $632.8 \mathrm{~nm}$ He-Ne laser. The transmission axis was set at an angle of $45^{\circ}$ with respect to the rubbing direction of the sample cell.

Figure 6 shows the characteristic curves of the voltage-dependent transmittance of the OCB cells before and after the atmospheric pressure plasma treatments. It can 


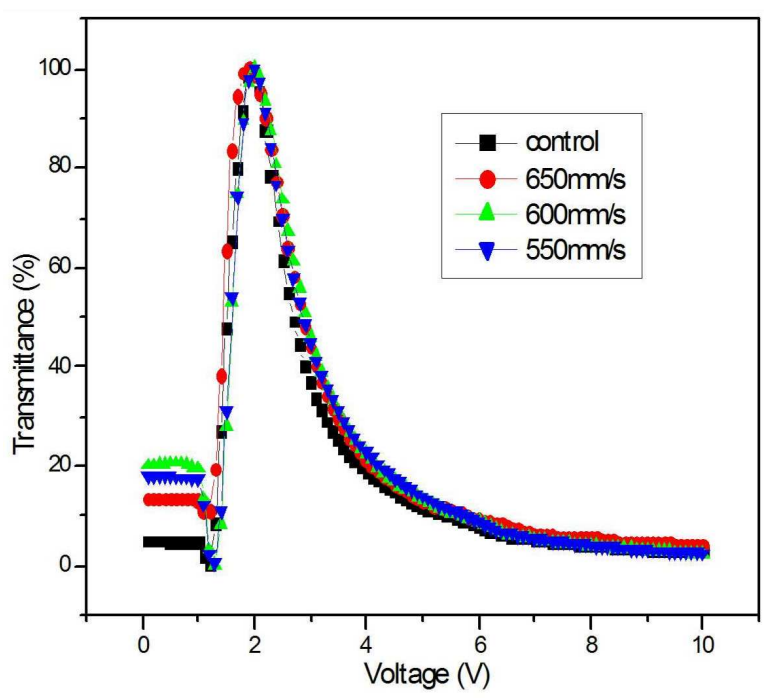

Fig. 6. Voltage-dependent transmittance curves for OCB cells. Critical voltage for the splay-to-bend alignment was around $2.0 \mathrm{~V}$ at elevation rate of $0.1 \mathrm{~V} / \mathrm{s}$.

be seen that the initial state of the splay alignment has been transformed to the bend state at the critical voltage of $2.0 \mathrm{~V}$. The further transition from the bright state (the maximum transmittance) to the dark state appeared in the $\pi$ cells with the increased voltage up to $10 \mathrm{~V}$.

\section{TABLE III}

Electro-optical properties of the OCB cells. Low response time characteristics were maintained at around $5 \mathrm{~ms}$.

\begin{tabular}{l|c|c|c|c}
\hline \hline \multicolumn{1}{c|}{ Sample } & Control & $650 \mathrm{~mm} / \mathrm{s}$ & $600 \mathrm{~mm} / \mathrm{s}$ & $550 \mathrm{~mm} / \mathrm{s}$ \\
\hline cell gap $[\mu \mathrm{m}]$ & 8.6 & 8.6 & 8.5 & 8.5 \\
pre-tilt angle $\left[{ }^{\circ}\right]$ & 6.6 & 6.5 & 6.5 & 6.4 \\
retardation $(\delta)$ & 365 & 389 & 410 & 404 \\
response time [ms] & 4.7 & 4.9 & 5.0 & 5.1
\end{tabular}

It has been noted that different initial transmittance was exhibited at the low voltage applied. Let us consider the electro-optical properties as shown in Table III. The light transmittance $(T)$ of the crossed polarizer can be written as

$$
T=(1 / 2)\left[\sin ^{2}(2 \phi) \sin ^{2}(\delta / 2)\right],
$$

where $\phi$ is the angle between the long axis of the liquid crystal molecules and the transmission axis of the polarizer. $\delta$ is indicated as the phase retardation. When the transmission axis was set at an angle of $45^{\circ}$, the brightest state could be achieved. Thus, the transmittance increased with the increasing phase retardation. The order of transmittance in the OCB cells would thus decrease as follows: $T_{600}>T_{650}>T_{550}>T_{\text {control. }}$. This is in accordance with the results of different initial transmittance in the $V-T$ curves. Furthermore, the data of the pre-tilt an- gles were nearly the same after the atmospheric pressure plasma treatments. The low response time characteristics were also maintained at around $5 \mathrm{~ms}$.

\section{Conclusions}

The atmosphere pressure plasma beam treatment process was successfully proposed to induce the partial liquid crystal alignment irregularity. It dramatically increased the number of the bend transition nucleation sites in the OCB display cells. The splay-to-bend transition and the warm-up time were thus significantly improved with the effective plasma beam treatments to the PI alignment films. This process has been very cost-effective, and could provide uniform treatment results. The processing parameters were also optimized at the plasma power of $700 \mathrm{~W}$, the plasma-to-substrate distance of $25 \mathrm{~mm}$, and $600 \mathrm{~mm} / \mathrm{s}$ plasma scan speed. The plasma bombardment angle has been set at zero. The number of the initial bend nucleation sites was greatly increased from 6 to 127 , and thus resulted in the reduction of transition time by up to $71 \%$ compared to the control sample. In addition, we could maintain the excellent electro-optical properties of the OCB cells, such as the quick response time of about $5 \mathrm{~ms}$.

\section{Acknowledgments}

This work was supported in part by the National Science Council (ROC) under NSC100-2221-E182-014.

\section{References}

[1] K. Kuboki, T. Miyashita, T. Isinabe, T. Uchida, Mol. Cryst. Liq. Cryst. 410, 391 (2004).

[2] C.L Kuo, T. Mifashita, M. Suzuki, T. Uchida, Appl. Phys. Lett. 68, 1461 (1996).

[3] S.W. Kim, L.C. Chien, Jpn. J. Appl. Phys. 43, 7643 (2004).

[4] G.M. Wu, H.W. Chien, J.W. Huang, H.L. Zeng, Nanotechnology 21, 134022 (2010).

[5] Y. Sun, H. Ma, Z. Li, Z. Zhang, R. Guan, Appl. Phys. Lett. 90, 091103 (2007).

[6] F. Ogasawara, K. Kuboki, K. Wako, T. Uchida, A. Yoshizawa, Jpn. J. Appl. Phys. 48, 051507 (2009).

[7] Y. Kizu, R. Hasegawa, Y. Kizaki, I. Amemiya, S. Uchikoga, in: SID Int. Symp. Dig. Tech. Papers, Eds. I. Kymissis, T. Fiske, Society for Information Display, Los Angeles 2008, p. 28.

[8] C.Y. Huang, R.X. Fung, Y.G. Lin, Jpn. J. Appl. Phys. 46, 5230 (2007).

[9] Y.K. Jang, P. Bos, Jpn. J. Appl. Phys. 46, 5821 (2007). 